\title{
Functional Subsystems and Quantum Redundancy in Photosynthetic Light Harvesting
}

\author{
Nolan Skochdopole and David A. Mazziott * \\ Department of Chemistry and The James Franck Institute, The University of Chicago, Chicago, IL 60637
}

(Dated: Submitted August 24, 2011; Published in J. Phys. Chem. Lett. 2011, 2, 2989)

\begin{abstract}
The Fenna-Matthews-Olson (FMO) antennae complex, responsible for light harvesting in green sulfur bacteria, consists of three monomers, each with seven chromophores. Here we show that multiple subsystems of the seven chromophores can transfer energy from either chromophore 1 or 6 to the reaction center with an efficiency matching or in many cases exceeding that of the full seven chromophore system. In the FMO complex these functional subsystems support multiple quantum pathways for efficient energy transfer that provide a built-in quantum redundancy. There are many instances of redundancy in nature, providing reliability and protection, and in photosynthetic light harvesting this quantum redundancy provides protection against the temporary or permanent loss of one or more chromophores. The complete characterization of functional subsystems within the FMO complex offers a detailed map of the energy flow within the FMO complex, which has potential applications to the design of more efficient photovoltaic devices.
\end{abstract}

\section{TABLE OF CONTENTS GRAPHIC}

After harvesting a photon of energy, the FennaMatthews-Olson (FMO) antennae complex transfers it to the reaction center.

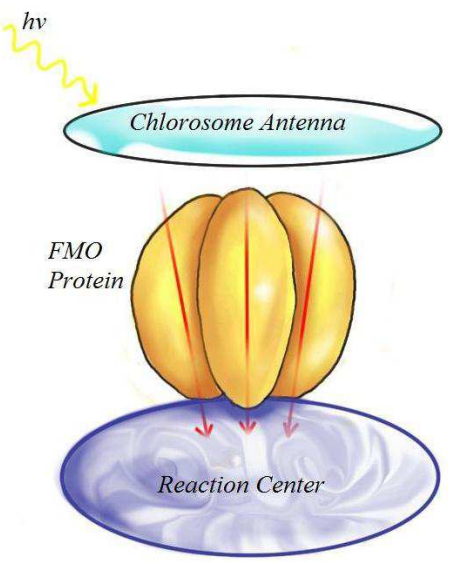

Keywords: functional subsystems, quantum redundancy, chromophores, photosynthetic light harvesting, Fenna-Matthews-Olson (FMO) antennae complex, entanglement, squared Euclidean distance

\section{LETTER}

Most photosynthetic organisms have light-harvesting antennae complexes composed of pigment molecules, called chromophores, whose electrons are excited by sunlight [1]. The excitation energy is transferred from the antennae complexes to reaction centers with nearly a $100 \%$ quantum efficiency [2]. For many years this transfer was thought to occur by a classical hopping mechanism, in which the excitation energy transfers from one chromophore to another in a downhill fashion until it reaches the reaction center [3, 4], but recently, new experimental evidence suggests that this transfer occurs by a quantum coherent mechanism [5, 6]. Quantum coherence in the Fenna-Matthews-Olson (FMO) antennae complex of green sulfur bacteria has been shown by twodimensional Fourier-transform electronic spectroscopy to last over $500 \mathrm{fs}$ at ambient temperatures and over $300 \mathrm{fs}$ at physiological temperatures $7-9]$.

In this Letter we show that photosynthetic light harvesting exhibits quantum redundancy. By quantum redundancy we mean that photosynthetic antennae complexes have multiple efficient quantum pathways for transferring energy to the reaction center. These pathways involve different subsets of the antennae's chromophores, which form functional subsystems. The antennae complex known as the FMO complex is a trimer where each monomer consists of seven bacteriochlorophyll-a chromophores [10]. Previous research has shown that in each monomer initial excitation can occur on either chromophore 1 or chromophore 6 and that this energy must move to chromophore 3 , which is most closely coupled with the reaction center [1]. Here we show that within each monomer of the FMO complex there exist many subsets of the seven chromophores with efficiencies close to or even better than the efficiency of the entire set of seven chromophores. Quantum redundancy contributes a robustness to nature's quantum device with potential survival benefits.

The computations presented here reveal that the functional subsystems achieve their efficiencies by a quantum mechanism similar to that of the full system including the roles of entanglement and environmental noise. We assess each subsystem's entanglement by a global entanglement measure 12, 13] based on the squared Euclidean distance [14 19]. The study of these chromophore subsystems gives more information about the role of each chromophore in the energy transfer in the whole FMO complex [20 31]. This more in-depth understanding can provide insight to other antennae complexes and ultimately be applied to create synthetic solar cells that rival the efficiency of nature. 
We consider a single monomer of the FMO complex with $M$ chromophore sites with the Hamiltonian

$$
\hat{H}=\sum_{j=1}^{M} \hbar \omega_{j} \sigma_{j}^{+} \sigma_{j}^{-}+\sum_{j \neq l} \hbar v_{j, l}\left(\sigma_{j}^{-} \sigma_{l}^{+}+\sigma_{j}^{+} \sigma_{l}^{-}\right),
$$

where $\sigma_{j}^{+}\left(\sigma_{j}^{-}\right)$creates (annihilates) a single excitation on chromophore $j$. The site energy of each chromophore is $\hbar \omega_{j}$ while the coupling constant between the pair of chromophores $j$ and $l$ is $\hbar v_{j, l}$.

The time evolution of the system's density matrix is governed by the quantum Liouville equation

$$
\frac{d}{d t} D=-\frac{i}{\hbar}[\hat{H}, D]+\hat{L}(D)
$$

where $D$ is the density matrix

$$
D=\sum_{k, l} \rho_{l}^{k}\left|\Psi_{k}\right\rangle\left\langle\Psi_{l}\right|
$$

$\rho_{l}^{k}$ are the elements of the density matrix $D$ in the basis set of wavefunctions $\left\{\Psi_{k}\right\}$, and $\hat{L}$ is the Lindblad operator, which accounts for interactions of the $M$ chromophores with the environment. At $t=0$ we initialize the density matrix with a single excitation (exciton) on either site 1 or 6 . Because the Hamiltonian does not mix singly excited wavefunctions with other wavefunctions, the set $\left\{\Psi_{k}\right\}$ only includes $M$ wavefunctions formed from sequentially considering a single excitation on each of the $M$ chromophores.

The Lindblad operator [30, 32] is the sum of three operators that account for dephasing, dissipation, and loss to the reaction center (sink)

$$
\hat{L}(D)=\hat{L}_{\mathrm{deph}}(D)+\hat{L}_{\mathrm{diss}}(D)+\hat{L}_{\mathrm{sink}}(D)
$$

where

$$
\begin{aligned}
\hat{L}_{\text {deph }}(D) & =\alpha \sum_{k} 2\langle k|D| k\rangle|k\rangle\langle k|-\{|k\rangle\langle k|, D\}, \\
\hat{L}_{\text {diss }}(D) & =\beta \sum_{k} 2\langle k|D| k\rangle|g\rangle\langle g|-\{|k\rangle\langle k|, D\}, \\
\hat{L}_{\text {sink }}(D) & =2 \gamma\langle 3|D| 3\rangle|s\rangle\langle s|-\gamma\{|3\rangle\langle 3|, D\} .
\end{aligned}
$$

The $|g\rangle$ denotes the state in which each of the $M$ chromophores is in its ground state, the $|k\rangle$ represent the $M$ excited states with each state having a single excitation on one of the $M$ chromophores, the state $|s\rangle$ denotes the reaction center (sink), and $|3\rangle$ indicates the excited state of the third chromophore multiplied by the ground states of the other $M-1$ chromophores. The particular form of the Lindblad operators chosen here follows the operators selected in previous studies of the FMO complex [30]. Further refinements of the system's interaction with the environment will not substantively affect the general conclusions made here about the role of functional subsystems within the FMO complex. The model incorporates two types of noise that affect the energy transfer. First, dissipation transfers energy from the chromophores back into the environment, wasting that energy. Second, dephasing dampens the coherence within the chromophore system. Finally, the Lindblad operator $\hat{L}_{\text {sink }}(D)$ is responsible for transferring energy from the third chromophore into reaction center, which acts as an energy sink and becomes a $M+1$ site in the density matrix. The rate parameters $\alpha, \beta$, and $\gamma$ were chosen in atomic units to be $1.52 \times 10^{-4}, 7.26 \times 10^{-5}$, and $1.21 \times 10^{-8}$, respectively. These values are consistent with those employed in Ref. [30].

Entanglement is a correlation that cannot occur in a classical system; Bell 33 defined entanglement as "a correlation that is stronger than any classical correlation." Parts of a quantum system become entangled when the total density matrix for the system cannot be expressed as a product of the density matrices for the parts [12, 13, 34]. Global entanglement [12, 13, 34] in the system is measured by the squared Euclidean distance between the density matrix $\rho$ and the nearest classical density matrix $\xi[14,19]$

$$
\sigma(\rho)=\sum_{i, j}\left|\rho_{j}^{i}-\xi_{j}^{i}\right|^{2} .
$$

In the basis of $M$ wavefunctions, in which each wavefunction has a single excitation on one of the $M$ chromophores, this measure corresponds to the sum of the squares of the off-diagonal elements of $\rho$, or the sum of the squares of the concurrences. The squared Euclidean distance is zero if and only if the excitations (excitons) on the chromophores are not entanglement. Both squared Euclidean distance and quantum relative entropy [29, 34], another measure of global entanglement, are Bergmann distances.

In Figs. 1-3 and Table I we study subsystems of the FMO complex, in which certain of the seven chromophores are "turned off" and the energy transfer is tracked within a smaller set of chromophores. For the full system of seven chromophores we employ the $7 \times 7$ Hamiltonian given in Ref. [30]; chromophores are removed from the full system by deleting rows and columns of the Hamiltonian matrix. We denote the subsystem by the numbers of the chromophores retained; for example, the subsystem with chromophores 1,2 , and 3 is denoted as 123 . Figure 1 shows the exciton populations of chromophores 1, 2, 3, and the reaction center for reduced system 123 and the full system. We observe that the two systems are quite similar, and yet they exhibit some important differences. The reduced system features a slightly slower decay of the exciton populations in chromophores 1 and 2, and most significantly, it transfers energy from chromophore 1 to the reaction center more quickly than the full system. Because chromophores 4-7 draw some of the excitation energy in the full system, the 

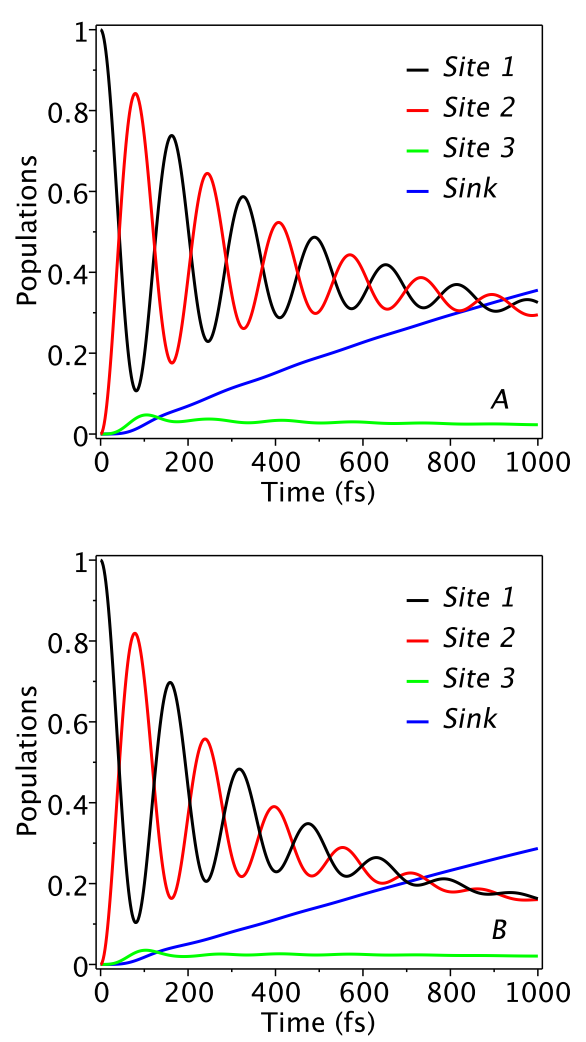

FIG. 1. Populations of chromophores 1-3 and the reaction center for (A) the subsystem 123 and (B) the full FMO system 1234567. Subsystem 123 of the FMO complex transfers energy more efficiently from chromophore 1 to the reaction center (sink) than the full FMO system.

populations of chromophores 1-3 and the reaction center are lower in the full system than in the reduced system.

The efficiency of energy transfer from site 1 to the sink is compared in Fig. 2 for several subsystems, 123, 1234,12345 (not shown because its efficiency is similar to 1234 ), and 123456, as well as the full system. We observe that subsystems 123,1234 , and 12345 are more efficient than the full system even though efficiency decreases as we add chromophores from 123 to 123456 . The addition of chromophores 4,5 , and 6 increases the number of sites for the energy to enter and thereby decreases the population of every site, including the reaction center. The addition of chromophore 7 to 123456 , however, improves efficiency; because chromophore 7 has a lower energy than chromophores 5 and 6 , it offers a new downhill quantum path through chromophore 4 , even lower in energy, to chromophore 3 and the reaction center. The importance of chromophore 7 can also be seen from Table I, which shows that the subsystem containing chromophores 12347 is the most efficient system, again because of the downhill path created from chromophore 7 to the reaction center.

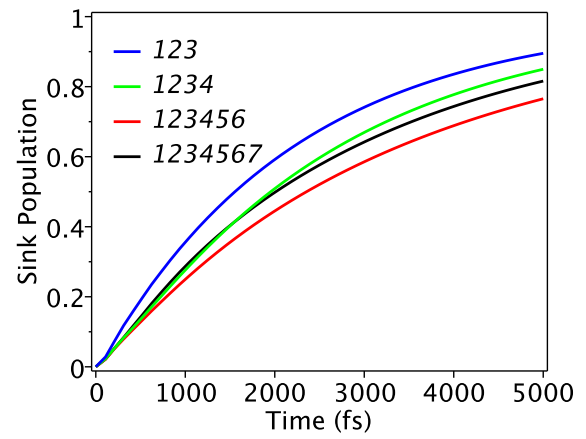

FIG. 2. Population in the reaction center for several chromophore subsystems. The subsystems 123, 1234, and 12345 (not shown) are more efficient than the full FMO system at transferring energy from chromophore 1 to the reaction center $(\sin \mathrm{k})$.

Table I reports the efficiencies of subsystems with the initial excitation on either chromophore 1 or chromophore 6. Subsystems with both chromophores 1 and 6 are included in both categories. The table shows that most of these subsystems are more efficient than the full system at transferring energy from either chromophore 1 or chromophore 6 to the reaction center. However, only one subsystem 123467 is more efficient than the full system when efficiency is assessed with equal weight given to the excitation pathways from chromophores 1 and 6 to the reaction center. Although this model suggests that chromophore 5 is superfluous, it may have other necessary duties in nature that are not considered in the present model. The superfluity of chromophore 5 is predictable from the Hamiltonian of the system, as the site energy for chromophore 5 is much higher than the other chromophores [11]. However, it is worth noting that this is only the case in the FMO complex for Prosthecochloris aestuarii, which we are studying in this Letter, while in the FMO complex for Chlorobium tepidum, chromophore 5 is not the highest in energy and its exclusion would most likely make the system less efficient. Very recent crystallographic and quantum chemistry studies 35, 36] indicate that there is likely an eighth chromophore in the FMO of Prosthecochloris aestuarii, which due to sample preparation is not present in the ultrafast spectroscopic studies [7 9]. An eighth chromophore would further support the existence and importance of functional subsystems with the seven chromophores in recent ultrafast experiments serving as a functional subsystem of the full system in nature.

The subsystems, we observe, function by a quantum mechanism similar to that observed in the full system. Two features of this mechanism, which have received significant attention for the full system in the literature 20 31], are: (i) dephasing from environmental noise and (ii) entanglement of excitons. As in the full system, the 
TABLE I. Populations of the reaction center of different chromophore subsystems at 2 ps.

\begin{tabular}{cccc}
\hline \hline & & \multicolumn{2}{c}{ Reaction Center Population } \\
\cline { 3 - 4 } Initial Excitation & System & No Dephasing & Dephasing \\
\hline Chromophore 1 & 123 & 0.387 & 0.592 \\
& 1234 & 0.161 & 0.509 \\
& 12345 & 0.144 & 0.506 \\
& 123456 & 0.120 & 0.445 \\
& 1234567 & 0.459 & 0.498 \\
& 12347 & 0.347 & 0.564 \\
Chromophore 6 6 & 123467 & 0.314 & 0.514 \\
& 346 & 0.010 & 0.090 \\
& 367 & 0.009 & 0.026 \\
& 3467 & 0.048 & 0.486 \\
& 3456 & 0.045 & 0.305 \\
& 34567 & 0.103 & 0.445 \\
& 234567 & 0.086 & 0.434 \\
& 1234567 & 0.139 & 0.433 \\
& 123467 & 0.074 & 0.456 \\
\hline \hline
\end{tabular}

dephasing assists transport of the excitons from chromophore 1 or 6 to the reaction center by facilitating the distribution of the exciton population from its initial chromophore. The efficiencies of different subsystems with and without dephasing are reported in Table I. The perceived enhancement is a characteristic of systems of heterogeneous chromophores, that is chromophores with different site energies. We also observe in Fig. 3, displaying the entanglement in several subsystems as well as the full system, that the entanglement of excitons exhibits similar behavior in the reduced systems as in the full system. We compute the entanglement to demonstrate that the transfer of energy for both reduced and full systems within the model occurs by the same quantum mechanism. (It is not the purpose of the present Letter to examine the relationship between entanglement and efficiency, which has been explored in previous work including a recent paper by one of the authors [37.) The addition of each chromophore slightly increases the total entanglement of the system, as measured by the squared Euclidean distance, by adding extra off-diagonal elements to the density matrix. Each system shows that the entanglement lasts for a few hundred femtoseconds, which matches previous experimental data as well as predictions from other theoretical models $[9,38]$.

Efficient photosynthetic light harvesting, according to recent experimental evidence, likely occurs by a quantum rather than a classical mechanism. In this Letter we have identified multiple functional subsystems of the FMO light-harvesting complex whose efficiencies are comparable to the efficiency of the full FMO complex. Many of these subsystems are, in fact, more efficient in transferring energy from either chromophore 1 or 6 to the reaction center, and one of these subsystems is even more efficient in transferring energy from both chromophores 1 and 6 to the reaction center. These functional subsystems

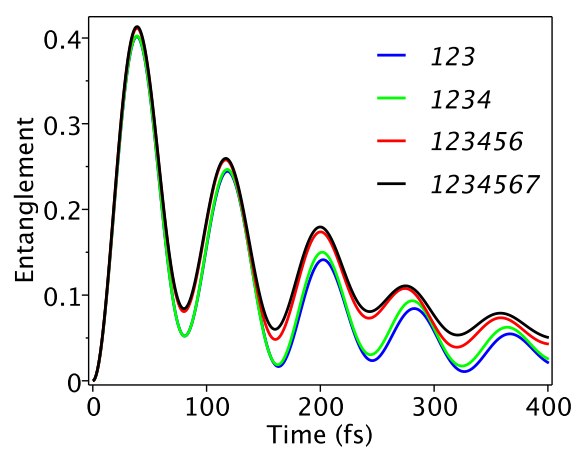

FIG. 3. Entanglement of excitons for several chromophore subsystems as a function of time. A measure of global entanglement is shown as a function of time (fs) for several chromophore subsystems, 123, 1234, and 12346, as well as the full system 1234567 . Like the full system, the subsystems exhibit entanglement of excitons between the chromophores.

provide evidence for quantum redundancy in photosynthetic light harvesting. There exists multiple quantum pathways or circuits that are capable of transferring energy efficiently to the reaction center. For example, subsystem 123 transfers energy from chromophore 1 to the reaction center with $59.2 \%$ efficiency (after $2 \mathrm{ps}$ ), and subsystem 3467 transfers energy from chromophore 6 to the reaction center with $48.6 \%$ efficiency. This built-in redundancy likely provides benefits to the natural system because damage to any chromophore save 3 will not disrupt light harvesting. The characterization of functional subsystems within the FMO complex offers a detailed map of the energy flow within the FMO complex with potential applications to the design of more efficient photovoltaic devices.

DAM gratefully acknowledges the National Science Foundation, the Army Research Office, Microsoft Corporation, the Henry and Camille Dreyfus Foundation, and the David and Lucile Packard Foundation for their generous support.

* damazz@uchicago.edu

[1] Blankenship, R. E. Molecular Mechanisms of Photosynthesis; Blackwell Science Ltd, 2002.

[2] Sension, R. J. "Biophysics: Quantum Path to Photosynthesis," Nature 2007, 446, 740-741.

[3] van Amerongen, H.; Valkunas, L.; van Grondelle, R. Photosynthetic Excitons; World Scientific Publishing Co. Pte. Ltd, 2000.

[4] Scholes, G. D. "Quantum-coherent Electron Energy Transfer: Did Nature Think of It First?," J. Phys. Chem. Lett. 2010, 1, 2-8.

[5] Cheng, Y. C.; Fleming, G. R. "Dynamics of Light Harvesting in Photosynthesis," Ann. Rev. Phys. Chem. 2009, 60, 241-262. 
[6] Brif, C.; Chakrabarti, R.; Rabitz, H. "Control of Quantum Phenomena: Past, Present and Future," New J. Phys. 2010, 12, 075008.

[7] Engel, G. S.; Calhoun, T. R.; Read, E. L.; Ahn, T. K.; Mancal, T.; Cheng, Y. C.; Blankenship, R. E.; Fleming, G. R. "Evidence For Wavelike Energy Transfer Through Quantum Coherence In Photosynthetic Systems," Nature 2007, 446, 782-786.

[8] Collini, E.; Wong, C. Y.; Wilk, K. E.; Curmi, P. M. G.; Brumer, P.; Scholes, G. D. "Coherently Wired LightHarvesting in Photosynthetic Marine Algae at Ambient Temperature," Nature 2010, 463, 644.

[9] Panitchayangkoon, G.; Hayes, D.; Fransted, K. A.; Caram, J. R.; Harel, E.; Wen, J.; Blackenship, R. E.; Engel, G. S. "Long-Lived Quantum Coherence in Photosynthetic Complexes at Physiological Temperature," Proc. Natl. Acad. Sci. 2010, 107, 12766-12770.

[10] Fenna, R. E.; Matthews, B. W. "Chlorophyll Arrangement in a Bacteriochlorophyll Protein From Chlorobium Limicola," Nature 1975, 258, 573-577.

[11] Adolphs, J.; Renger, T. "How Proteins Trigger Excitation Energy Transfer in the FMO Complex of Green Sulfur Bacteria," Biophys. J. 2006, 91, 2778-2797.

[12] Huang, Z.; Kais, S. "Entanglement as a Measure of Electron-Electron Correlation in Quantum Chemistry Calculations," Chem. Phys. Lett. 2005, 413, 1-5.

[13] Kais, S. "Entanglement, Electron Correlation, and Density Matrices," Adv. Chem. Phys. 2007, 134, 493.

[14] Pittenger, A. O.; Rubin, M. H. "Convexity and the Separability Problem of Quantum Mechanical Density Matrices," Lin. Alg. Appl. 2002, 346, 47-71.

[15] Bertlmann, R. A.; Narnhofer, H.; Thirring, W. "Geometric Picture of Entanglement and Bell Inequalities," Phys. Rev. A 2002, 66, 032319.

[16] Greenman, L.; Mazziotti, D. A. "Strong Electron Correlation in the Decomposition Reaction of Dioxetanone with Implications for Firefly Bioluminescence," J. Chem. Phys. 2010, 133, 164110.

[17] Juhasz, T.; Mazziotti, D. A. "The Cumulant TwoParticle Reduced Density Matrix as a Measure of Electron Correlation and Entanglement," J. Chem. Phys. 2006, 125, 174105.

[18] Mazziotti, D. A.; Herschbach, D. R. "Boson Correlation Energies and Density Matrices From Reduced Hamiltonian Interpolation," Phys. Rev. A 2000, 62, 043603.

[19] Harriman, J. E. "Geometry of Density Matrices .1. Definitions, N Matrices and 1 Matrices," Phys. Rev. A 1978, 17, 1249-1256.

[20] Zhu, J.; Kais, S.; Rebentrost, P.; Aspuru-Guzik, A. "Modified Scaled Hierarchical Equation of Motion Approach for the Study of Quantum Coherence in Photosynthetic Complexes," J. Phys. Chem. B 2011, 115, 1531-1537.

[21] Huo, P. F.; Coker, D. F. "Theoretical Study of Coherent Excitation Energy Transfer in Cryptophyte Phycocyanin 645 at Physiological Temperature," J. Phys. Chem. Lett. 2011, 2, 825-833.

[22] Kelly, A.; Rhee, Y. M. "Mixed Quantum-Classical Description of Excitation Energy Transfer in a Model Fenna-Matthews-Olsen Complex," J. Phys. Chem. Lett.
2011, 2, 808-812.

[23] Mancal, T.; Balevicius, V.; Valkunas, L. "Decoherence In Weakly Coupled Excitonic Complexes," J. Phys. Chem. A 2011, 115, 3845-3858.

[24] Sener, M.; Strumpfer, J.; Hsin, J.; Chandler, D.; Scheuring, S.; Hunter, C. N.; Schulten, K. "Forster Energy Transfer Theory as Reflected in the Structures of Photosynthetic Light-Harvesting Systems," Chem. Phys. Chem. 2011, 12, 518-531.

[25] Bradler, K.; Wilde, M. M.; Vinjanampathy, S.; Uskov, D. B. "Identifying the Quantum Correlations in Light-harvesting Complexes," Phys. Rev. A 2010, 82, 062310.

[26] Liang, X. T. "Excitation Energy Transfer: Study with Non-Markovian Dynamics," Phys. Rev. E 2010, 82, 051918.

[27] Nalbach, P.; Thorwart, M. "Quantum Coherence and Entanglement in Photosynthetic Light-Harvesting Complexes," In Quantum Coherence and Entanglement in Photosynthetic Light-Harvesting Complexes; 2010; Vol. 83, pp 39-75.

[28] Novoderezhkin, V. I.; van Grondelle, R. "Origins and Models of Energy Transfer in Photosynthetic LightHarvesting," Phys. Chem. Chem. Phys. 2010, 12, 73527365 .

[29] Sarovar, M.; Ishizaki, A.; Fleming, G. R.; Whaley, K. B. "Quantum Entanglement in Photosynthetic Light-Harvesting Complexes," Nature Physics 2010, 6, 462-467.

[30] Chin, A. W.; Datta, A.; Caruso, F.; Huelga, S. F.; Plenio, M. B. "Noise-Assisted Energy Transfer in Quantum Networks and Light-Harvesting Complexes," New J. Phys. 2010, 12, 065002.

[31] Wu, J. L.; Liu, F.; Shen, Y.; Cao, J. S.; Silbey, R. J. "Efficient Energy Transfer in Light-Harvesting Systems, I: Optimal Temperature, Reorganization Energy and Spatial-temporal Correlations," New J. Phys. 2010, 12, 105012.

[32] Lindblad, G. "On the Generators of Quantum Dynamical Semigroups," Commun. Math. Phys. 1976, 48, 119.

[33] Bell, J. S. "On the Einstein-Podolsky-Rosen Paradox," Physics 1964, 1, 195-200.

[34] Horodecki, R.; Horodecki, P.; Horodecki, M.; Horodecki, K. "Quantum Entanglement," Rev. Mod. Phys. 2009, 81, 865-942.

[35] am Busch, M. S.; Müh, F.; Madjet, M. E.; Renger, T. "Eighth Bacteriochlorophyll Completes the Excitation Energy Funnel in the FMO Protein," J. Phys. Chem. Lett. 2011, 2, 93.

[36] Tronrud, D. E.; Wen., J. Z.; Gay, L.; Blankenship, R. E. "Structural Basis for the Difference in Absorbance Spectra for the FMO Antenna Protein from Various Green Sulfur Bacteria," Photosynth. Res. 2009, 100, 79-87.

[37] Mazziotti, D. A. unpublished.

[38] Ishizaki, A.; Fleming, G. R. "Theoretical Examination of Quantum Coherence in a Photosynthetic System at Physiological Temperature," Proc. Nat. Acad. Sci. 2009, 106, 17255-17260. 